\title{
CCD photometry, spin and shape model of the asteroid 1572 Posnania*
}

\author{
T. Michałowski ${ }^{1}$, W. Pych ${ }^{2}$, T. Kwiatkowski ${ }^{1}$, A. Kryszczyńska ${ }^{1}$, P. Pravec ${ }^{3}$, W. Borczyk ${ }^{1}$, A. Erikson ${ }^{4}$, \\ W. Wisniewski ${ }^{5, \star \star}$, F. Colas $^{6}$, and J. Berthier ${ }^{6}$ \\ 1 Astronomical Observatory, A. Mickiewicz University, Słoneczna 36, 60-286 Poznań, Poland \\ 2 Copernicus Astronomical Center, Bartycka 18, 00-716 Warszawa, Poland \\ 3 Astronomy Institute of the Academy of Sciences, Ondrejov, Czech Republic \\ ${ }^{4}$ Institute of Space Sensor Technology and Planetary Exploration, Rutherford str. 2, 12489 Berlin, Germany \\ ${ }^{5}$ Lunar and Planetary Laboratory, University of Arizona, Tucson, Arizona 85721, USA \\ 6 Institut de Mecanique Celeste, 77 Av. Denfert Rochereau, 75014 Paris, France
}

Received 19 January 2001 / Accepted 17 March 2001

\begin{abstract}
Lightcurves of the asteroid 1572 Posnania were obtained at six observatories on 19 nights during four apparitions: in 1993, 1994-95, 1996, and 1999. The synodical period is found to be 8.048 hours and the observed amplitudes of brightness variations are in the range of $0.37-0.40$ mag. The sense of rotation is retrograde with a sidereal period $P_{\text {sid }}=0.3353931 \pm 0.0000003$ days. The ecliptic coordinates of the pole of this asteroid are $\lambda_{\mathrm{p}}=46^{\circ} \pm 6^{\circ}$ and $\beta_{\mathrm{p}}=-65^{\circ} \pm 5^{\circ}$. The shape of Posnania can be approximated by a triaxial ellipsoid model with semiaxes ratios $a / b=1.35 \pm 0.01$ and $b / c=1.04 \pm 0.03$.
\end{abstract}

Key words. techniques: photometric - minor planets: asteroids

\section{Introduction}

Earth-based observations, especially CCD photometry, provide the most abundant data on the physical properties of the asteroid population. Rotational periods, orientations of spin vectors and triaxial ellipsoid models can be determined from the brightness variation of the asteroids as they spin about their axes. A review of such techniques was given by Magnusson et al. (1989). The method proposed by Michałowski (1993) has been used in the present work.

The photometric database contains lightcurves of more than 700 asteroids, but spin vectors have been determined only for about 100 of them. The best known spin and shape database has been compiled by Per Magnusson and can be found in The Small Bodies Node of the NASA Planetary Data System (http://pdssbn. astro.umd.edu/) or in Uppsala Observatory (http://www. astro.uu.se).

In order to enlarge the sample of asteroids with known shapes and spin vectors, we have carried out CCD observations of the main belt asteroid 1572 Posnania. Our choice

\footnotetext{
Send offprint requests to: T. Michałowski,

e-mail: tmich@amu.edu.pl

* Partly based on observations collected at the European Southern Observatory, La Silla, Chile.

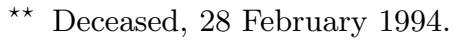

of this particular object was motivated by the fact that it was discovered about 50 years ago in Poznań. Prior to the present paper no lightcurves have been reported for this asteroid in the literature and the rotational period, the spin vector and the shape model have been unknown.

On 22 September 1949, two astronomers J. Dobrzycki and A. Kwiek from the Poznań Astronomical Observatory (Poland), discovered a new minor planet. They named it Posnania in honour of the place of the discovery. Following this tradition, a group of people from Poznań decided to study this asteroid by using CCD photometry. They were joined by colleagues from five other observatories and the results of their combined effort are described in this paper.

The asteroid 1572 Posnania was studied by the IRAS satellite and its diameter $(33.6 \mathrm{~km})$ and albedo $(0.156)$ have been determined

(http://pdssbn.astro.umd.edu/).

\section{Observations}

Asteroid 1572 Posnania was observed on 19 nights during four oppositions: 1993, 1994-95, 1996, and 1999. The majority of the data were obtained at Ostrowik (Warsaw University Observatory, Poland), Pic du Midi (France) and Borowiec (Poznań Astronomical Observatory, Poland) where $0.60 \mathrm{~m}, 1.05 \mathrm{~m}$, and $0.40 \mathrm{~m}$ telescopes, respectively, were used. All were equipped 
with CCD cameras, and all details concerning the instruments and the reduction procedure were described by Michałowski et al. (2000).

On four nights in September 1993 Posnania was observed at Ondrejov Observatory (Czech Republic). The measurements were performed using a small $0.18 \mathrm{~m}$ reflector and SBIG ST-6 camera with the $R$ filter (Pravec et al. 1994). This is the same instrument which had been used at Ondrejov during an international observational campaign for the asteroid 4179 Toutatis in 1992-93 (Spencer et al. 1995).

1572 Posnania was also observed at Mt. Lemmon (Arizona, USA) on 14 October 1993. The $1.50 \mathrm{~m} \mathrm{NASA-}$ $U A$ telescope and a Hamamatsu $\mathrm{GaAs}(\mathrm{Cs})$ photomulitiplier plus 8-colour filters were used (see Wisniewski et al. 1997). These measurements were reduced to the $V$ Johnson-Morgan standard system.

At ESO (La Silla, Chile), Posnania was observed with the $0.61-\mathrm{m}$ Bochum telescope equipped with the DLR MK II CCD camera. The photometric reduction was performed with the ASTPHOT package developed by S. Mottola at DLR. These data were transformed to the standard $V$ magnitude using stars from Laskar et al. (1988).

The aspect data of the asteroid are listed in Table 1 . The columns give the date of the observation referring to the mid-time of the observed lightcurve, asteroid-Sun $(r)$ and asteroid-Earth $(\Delta)$ distances (in AU), solar phase angle, as well as ecliptic longitude $(\lambda)$ and latitude $(\beta)$ for the J2000 epoch. The names of the observatories as mentioned above are listed in the last column of the table.

Our observations are presented as composite lightcurves (Figs. 1-4) which have been constructed with a procedure described in Magnusson \& Lagerkvist (1990). The synodical period is shown in each graph. A vertical position of each lightcurve was obtained by minimizing the dispersion of data points relative to their neighbours. The abscissae are the rotational phases with zero points corrected for light-time.

In September-October 1993, the asteroid was observed on 10 nights (Table 1). These data have allowed us to determine the synodical period of 8.048 hours, which was used to construct a composite lightcurve presented in Fig. 1. It shows rather irregular light variations, with different levels of maxima and minima, respectively and the amplitude of $0.38 \mathrm{mag}$.

During the next opposition Posnania was observed from November 1994 to February 1995. The irregular lightcurve (Fig. 2) confirmed the synodical period of 8.048 hours and displayed the amplitude of $0.40 \mathrm{mag}$.

Figure 3 presents the results of the observations carried out in January-February 1996. The composite lightcurve with the amplitude of $0.38 \mathrm{mag}$ is very similar in shape to that from the 1993 apparition, as during these two oppositions Posnania was observed from opposite directions.

The last observations were performed in October and November 1999. The composite lightcurve confirmed the synodical period of 8.048 hours and the amplitude of brightness variation was 0.37 mag (Fig. 4).
As is seen from the above description, the amplitudes of Posnania's lightcurves obtained during four different oppositions are in the range of $0.37-0.40 \mathrm{mag}$. The small differences in the amplitudes indicate that the axis of rotation of Posnania is far from the ecliptic plane.

\section{Spin axis and triaxial ellipsoid model of $\mathbf{1 5 7 2}$ Posnania}

In the method proposed by Michałowski (1993) the magnitudes, amplitudes, and epochs of the maxima are used to determine spin vector, sidereal period, and triaxial ellipsoid model of a given asteroid. The results are obtained by building a set of nonlinear equations whose solution is found by the least square fitting.

The observed amplitudes of Posnania have been reduced to zero phase angle with the amplitude-phase relationship described by Zappala et al. (1990). The correction coefficient was found to be $m=0.006$.

As only the ESO and Mt. Lemmon observations (obtained on three nights) were reduced to the standard system, we have not been able to reduce the observed magnitudes to the zero phase angle. Instead we have used the average value of $G=0.15$ for the $H G$ - magnitude system (Bowell et al. 1989).

It is usually assumed that lightcurves from at least three different oppositions of a main belt asteroid are required to derive its pole and shape. In our case, we have data from four oppositions, but they cover only a half of Posnania's orbit around the Sun. Moreover, the ecliptic longitudes of the asteroids in 1993 and 1996 differ by about $180^{\circ}$. It is not a good situation because of the two-fold ambiguity of an asteroid pole (see Magnusson et al. 1989; Michałowski 1993; Michałowski et al. 2000 for details).

Using all lightcurves from four oppositions reported here we have derived physical parameters for Posnania. The sense of rotation of this asteroid is found to be retrograde. The sidereal period, the ecliptic coordinates of the asteroid north pole, the triaxial ellipsoid shape, and their formal errors are as follows:

$$
\begin{aligned}
P_{\text {sid }} & =0.3353931 \pm 0.0000003 \text { (days) } \\
\lambda_{\mathrm{p}} & =46^{\circ} \pm 6^{\circ} \\
\beta_{\mathrm{p}} & =-65^{\circ} \pm 5^{\circ} \\
a / b & =1.35 \pm 0.01 \\
b / c & =1.04 \pm 0.03 .
\end{aligned}
$$

The four oppositions when we observed Posnania did not cover the whole orbit of the asteroid around the Sun. It is probably the reason that we have not obtained a unique solution for the sidereal period. A second model with a period of $0.3352664 \pm 0.0000004$ days is less probable but should be considered. Further observations are needed to resolve this ambiguity.

The mean diameter of Posnania, as obtained by the IRAS satellite, is $D=33.6 \pm 2.5 \mathrm{~km}$ (http://pdssbn.astro.umd.edu/). The spin vector of 
Table 1. Aspect data for 1572 Posnania

\begin{tabular}{|c|c|c|c|c|c|c|}
\hline \multirow[t]{2}{*}{ Date (UT) } & \multirow{2}{*}{$\begin{array}{c}r \\
(\mathrm{AU})\end{array}$} & \multirow{2}{*}{$\begin{array}{c}\Delta \\
(\mathrm{AU})\end{array}$} & \multirow{2}{*}{$\begin{array}{c}\text { Phase } \\
\text { angle } \\
\left({ }^{\circ}\right)\end{array}$} & \multicolumn{2}{|c|}{$\lambda_{(\mathrm{J} 2000)} \beta$} & \multirow[t]{2}{*}{ Obs. } \\
\hline & & & & $\left({ }^{\circ}\right)$ & $\left({ }^{\circ}\right)$ & \\
\hline \multicolumn{7}{|l|}{1993} \\
\hline Sep. 19.0 & 2.4544 & 1.4550 & 3.09 & 3.27 & -2.33 & Ond \\
\hline Sep. 20.0 & 2.4543 & 1.4538 & 2.62 & 3.06 & -2.22 & Ond \\
\hline Sep. 21.0 & 2.4543 & 1.4528 & 2.16 & 2.85 & -2.11 & Ond \\
\hline Sep. 23.0 & 2.4542 & 1.4516 & 1.27 & 2.43 & -1.90 & Ond, Ost \\
\hline Sep. 24.0 & 2.4541 & 1.4514 & 0.91 & 2.22 & -1.79 & Ost \\
\hline Sep. 25.0 & 2.4541 & 1.4515 & 0.69 & 2.01 & -1.68 & Ost \\
\hline Sep. 26.0 & 2.4541 & 1.4519 & 0.78 & 1.80 & -1.57 & Ost \\
\hline Oct. 14.2 & 2.4557 & 1.5051 & 8.99 & 358.26 & 0.42 & Lem \\
\hline Oct. 18.9 & 2.4567 & 1.5325 & 10.98 & 357.57 & 0.90 & Ost \\
\hline Oct. 19.9 & 2.4569 & 1.5387 & 11.36 & 357.45 & 0.99 & Ost \\
\hline \multicolumn{7}{|l|}{ 1994-95 } \\
\hline Nov. 14.1 & 3.0649 & 2.5583 & 17.46 & 118.93 & 15.94 & Pic \\
\hline Dec. 04.0 & 3.1059 & 2.3714 & 13.92 & 118.50 & 17.34 & Ost \\
\hline Feb. 04.0 & 3.2293 & 2.3519 & 9.34 & 107.19 & 17.40 & Ost \\
\hline \multicolumn{7}{|l|}{1996} \\
\hline Jan. 17.2 & 3.7014 & 3.1259 & 13.47 & 177.35 & 6.08 & Pic \\
\hline Feb. 17.3 & 3.7205 & 2.8139 & 6.97 & 174.46 & 5.70 & ESO \\
\hline Feb. 24.2 & 3.7242 & 2.7751 & 5.00 & 173.26 & 5.53 & ESO \\
\hline \multicolumn{7}{|l|}{1999} \\
\hline Oct. 31.0 & 2.6922 & 1.8687 & 14.21 & 76.13 & 16.13 & Bor \\
\hline Nov. 04.0 & 2.6996 & 1.8468 & 13.03 & 75.65 & 16.54 & Bor \\
\hline Nov. 16.0 & 2.7222 & 1.8026 & 9.43 & 73.66 & 17.62 & Bor \\
\hline
\end{tabular}

Observatory code: Ond - Ondrejov; Ost - Ostrowik; Lem - Mt Lemmon; Pic - Pic du Midi; ESO - European Southern Observatory; Bor - Borowiec.

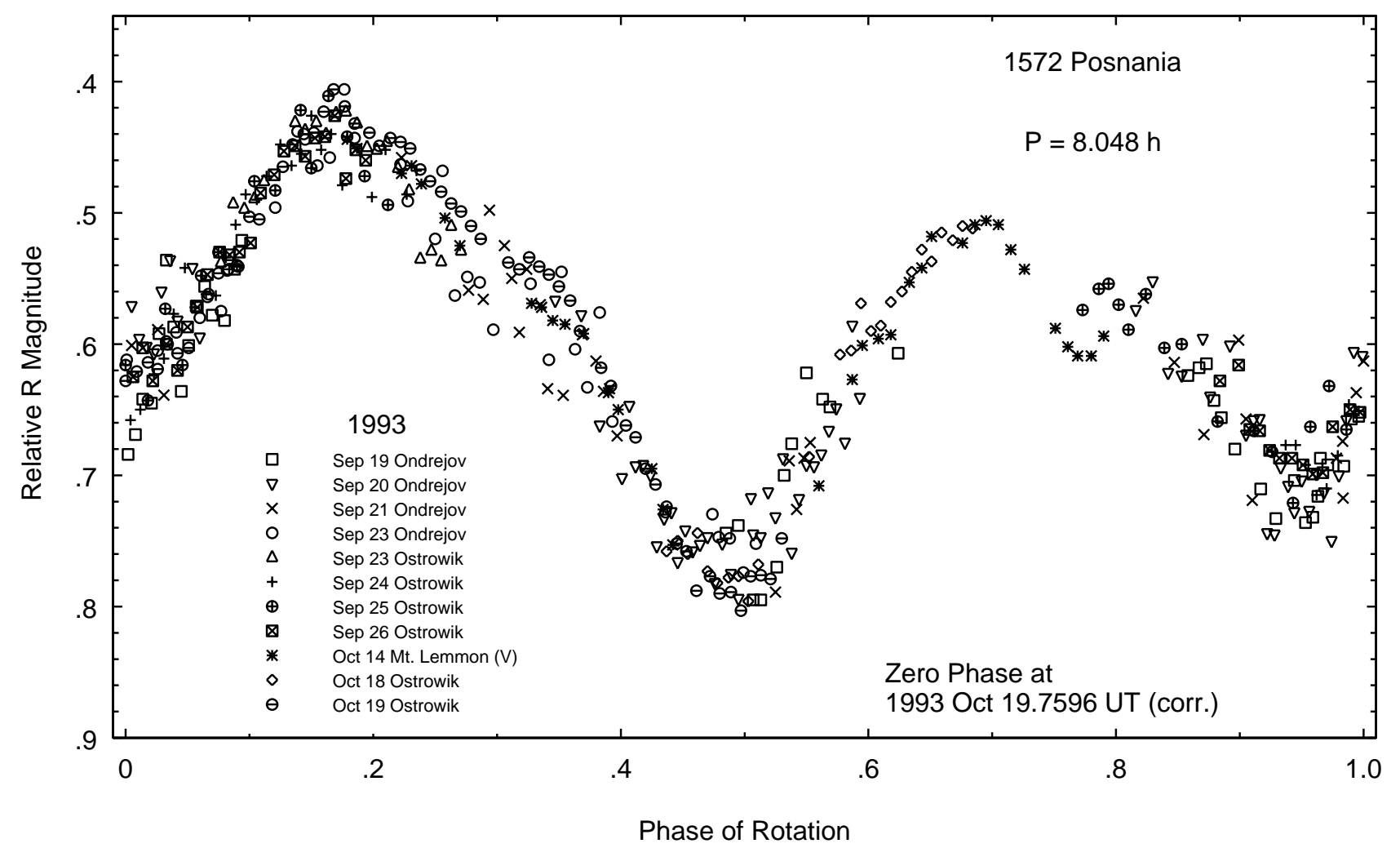

Fig. 1. Composite lightcurve of 1572 Posnania in 1993 


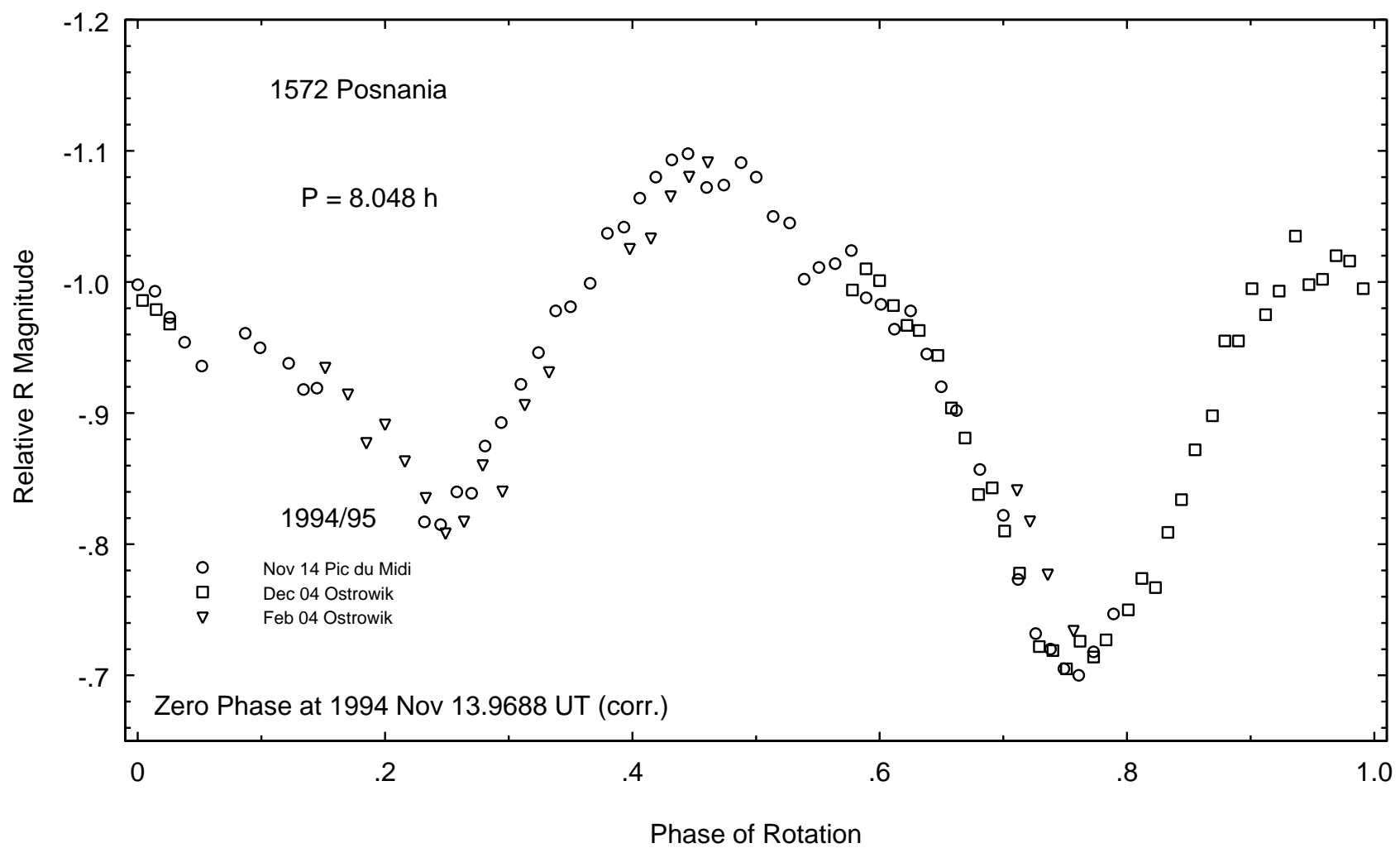

Fig. 2. Composite lightcurve of 1572 Posnania in 1994-95

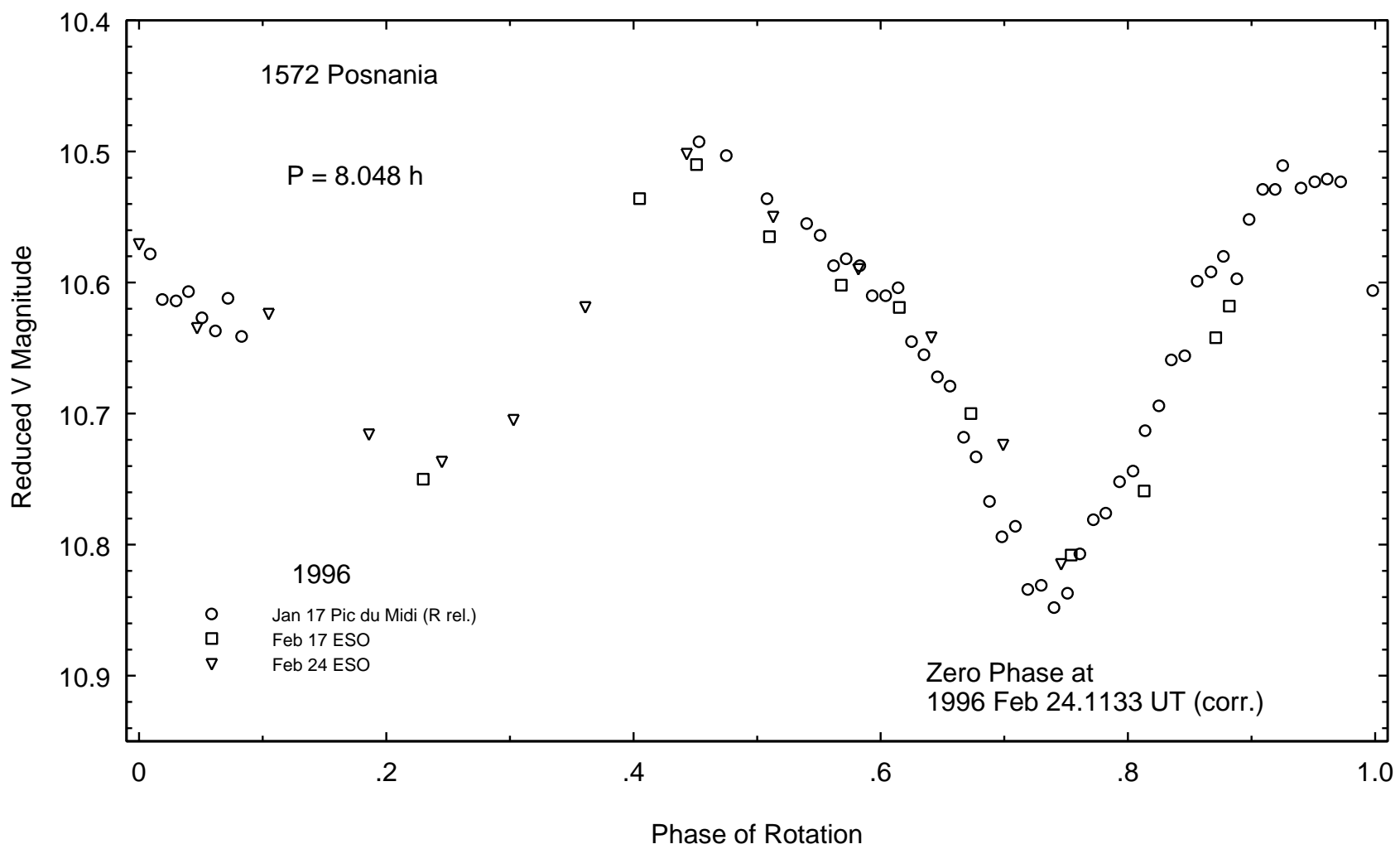

Fig. 3. Composite lightcurve of 1572 Posnania in 1996. The points from 17 Feb. are shifted by -0.066 mag with respect to those from 24 Feb. 


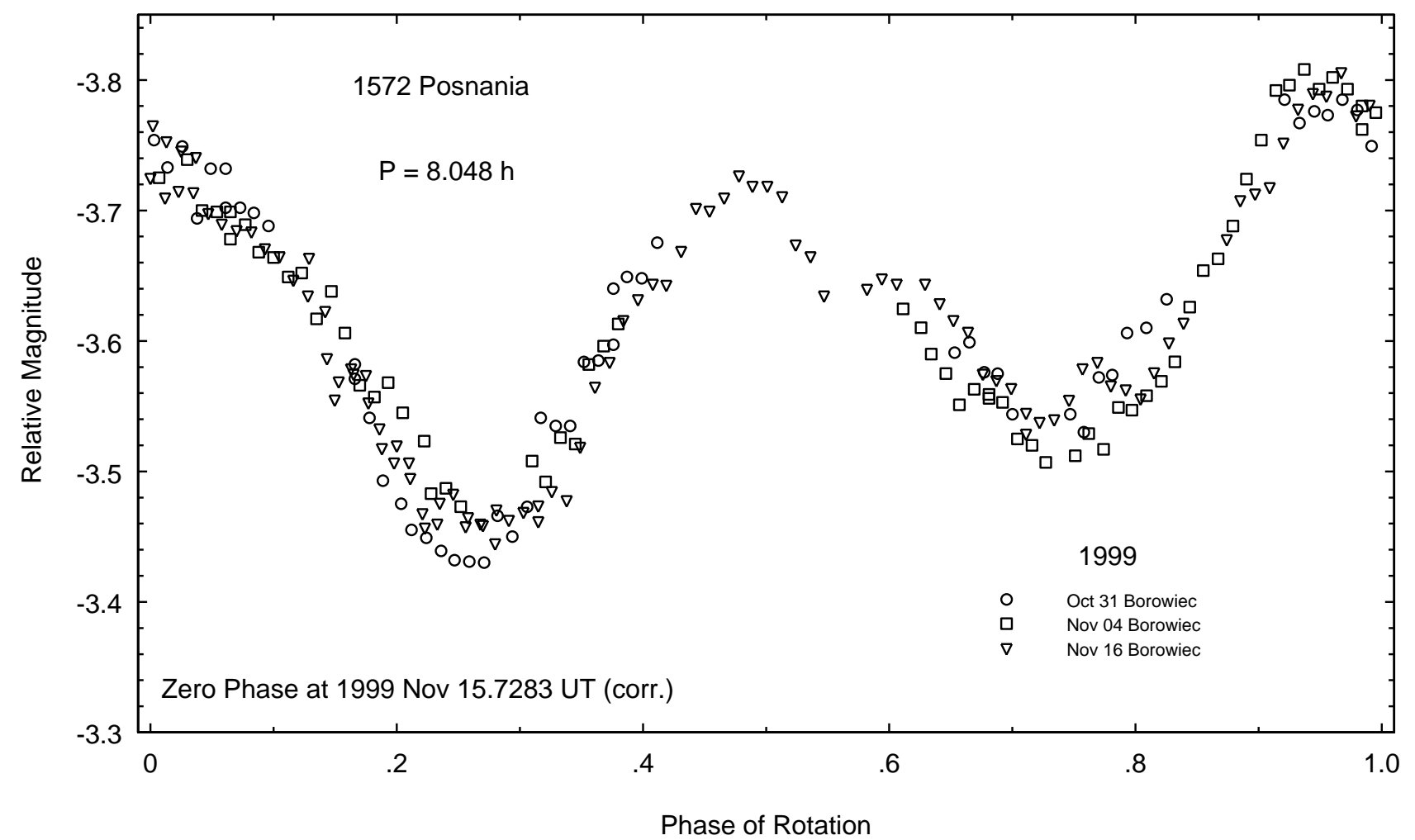

Fig. 4. Composite lightcurve of 1572 Posnania in 1999

Posnania shows that we observed this asteroid close to equatorial aspects during all oppositions. In such cases an area of the cross-section of the triaxial ellipsoid model varied from $\pi a c$ to $\pi b c$. We can assume that similar aspects were during the IRAS measurements and the area of the cross-section of the reported sphere is equal to the mean area of the cross-section of the triaxial ellipsoid model as obtained in the present paper $\left(\pi(D / 2)^{2}=\pi c(a+b) / 2\right)$. This assumption allowed us to obtain the following values for the major axes of Posnania: $2 a \times 2 b \times 2 c=$ $(42.6 \pm 5.4) \times(31.6 \pm 3.8) \times(30.4 \pm 2.8) \mathrm{km}^{3}$.

The results for the spin and shape model of 1572 Posnania can be improved, especially for the sidereal period. New observations should be carried out when the asteroid takes different orientations with respect to the Earth. The most promising are the oppositions in April 2002 and June 2003 when the ecliptic longitudes of Posnania are about $200^{\circ}$ and $260^{\circ}$, respectively. However, these data should be collected at southern observatories, especially in 2003 when the declination of the asteroid is about $-40^{\circ}$.

Acknowledgements. This work was partially supported by the Polish KBN Grant 2 P03D 007 18. AE gratefully thanks S. Mottola for letting him use the reduction program AST PHOT. The Borowiec observations were reduced with the CCLRS STARLINK package.

\section{References}

Bowell, E., Hapke, B., Domingue, D., et al. 1989, in Asteroids II (Univ. of Arizona Press, Tucson), 524

Laskar, B. M., et al. 1988, ApJS, 68, 1

Magnusson, P., \& Lagerkvist, C.-I. 1990, A\&AS, 86, 45

Magnusson, P., Barucci, M. A., Drummond, J. D., et al. 1989, in Asteroids II (Univ. of Arizona Press, Tucson), 66

Michalowski, T. 1993, Icarus, 106, 563

Michalowski, T., Pych, W., Berthier, J., et al. 2000, A\&AS, 146,471

Pravec, P., et al. 1994, Exper. Astron., 5, 375

Spencer, J. R., Akimov, L. A., Angeli, C., et al. 1995, Icarus, 117,71

Wisniewski, W. Z., Michalowski, T., Harris, A. W., \& McMillan, R. S. 1997, Icarus, 126, 395

Zappala, V., Cellino, A., Barucci, M. A., et al. 1990, A\&A, 231, 548 